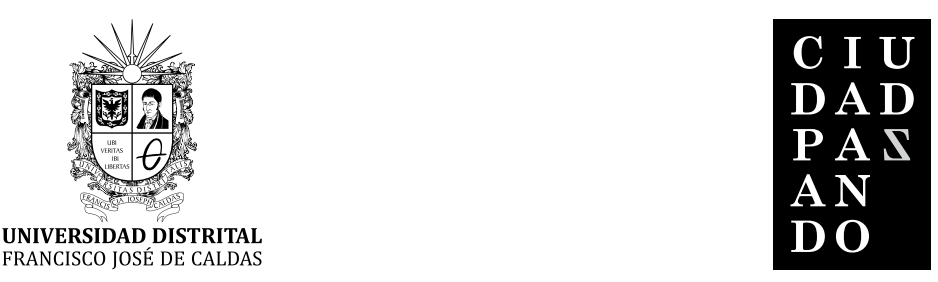

DOSSIER

Artículo de investigación científica

\title{
La investigación acción participante: una oportunidad para la transformación de la cátedra de paz en la universidad ${ }^{1}$
}

The investigation participating action: an opportunity for the transformation of the chair of peace in the university

A ação participante da investigação: uma oportunidade para a transformação da cadeira de paz na universidade

\section{Francy Yamile Tatar Garnica ${ }^{2}$}

\section{Jorge Enrique Vargas González ${ }^{3}$}

Para citar este artículo: Tatar, F. y Vargas, J. (2017). La investigación acción participante: una oportunidad para la transformación de la cátedra de paz en la universidad. Revista Ciudad Paz-ando, 10(2), 40-53.

doi: https://doi.org/10.14483/2422278X.12716

Fecha de recepción: 13 de noviembre de 2017

Fecha de aprobación: 16 de febrero de 2018

\footnotetext{
1 Avances de proyecto de investigación en curso: “Los jóvenes universitarios se transforman en constructores de paz". Doctorado en Ciencias Sociales Niñez y Juventud, Universidad Manizales - CINDE. Línea de investigación: Política Pública y Programas en niñez y Juventud.

2 Estudiante del Doctorado en Ciencias Sociales Niñez y Juventud, Universidad Manizales - CINDE. Docente Catedrática de la Fundación Universitaria Los Libertadores, Colombia. Correo electrónico: fytatarg@libertadores.edu.co

3 Doctor en Ciencias Sociales, Niñez y Juventud de la Universidad de Manizales - CINDE. Director en la Línea Políticas Públicas del Doctorado en Ciencias Sociales, Niñez y Juventud de la Universidad de Manizales, Colombia. Correo electrónico: jorvargas@gmail.com
} 


\section{RESUMEN}

Este artículo es una revisión de los principios fundamentales de la investigación acción participante (IAP) en el marco del paradigma sociocrítico. Aborda la fundamentación conceptual de esta metodología, su alcance y los procedimientos usuales desde la perspectiva de sus representantes teóricos más significativos. Investigar desde el paradigma sociocrítico implica intrínsecamente un compromiso político, social, por la justicia, la equidad y la paz. Desde la historia de la IAP, se verá que este método implica la promoción de transformaciones sociales o educativas, por lo cual es definido como un proceso que no solo busca comprender y generar conocimiento, sino que también espera suscitar cambios cultivados por y con los actores involucrados. Sus principios exaltan la naturaleza inductiva, dialógica e ideográfica del conocimiento; además, establecen una relación inseparable de la teoría y la práctica. Los procedimientos planteados por diferentes autores siempre implican fases recursivas de acción-reflexión-acción, un bucle abierto en permanente movimiento. A la postre, se verá que este tipo de investigación representa una oportunidad para la transformación de la cátedra de paz, lo que permitirá visualizar las posibles aplicaciones del método en el contexto universitario.

Palabras clave: generación de conocimiento, investigación acción participativa, transformación social.

This article is a review of the fundamental principles of Participatory Action Research (PAR) within the framework of the socio-critical paradigm. It addresses the conceptual foundation of this methodology, scope and the usual procedures from the perspective of its most significant theoretical representatives. Investigating from the socio-critical paradigm intrinsically implies a political, social commitment, for justice, equality and peace. From the history of the PAR, this method involves the promotion of social or educational transformations, so it is a process that not only seeks and generates knowledge, but also hopes to arouse changes cultivated by and with the actors involved. Its principles exalt the inductive, dialogical and ideographic nature of knowledge; in addition, an inseparable relationship between theory and practice. The procedures proposed by different authors, always involve the recursive phases of action-reflection-action, an open loop in permanent movement. In the end we will see that this type of research represents an opportunity for the transformation of the chair of peace, which will allow to visualize the possible applications of the method in the university context.

Keywords: knowledge generation, participatory action research, social transformation.
Este artigo é uma revisão dos princípios fundamentais da Pesquisa de Ação Participativa (PAP) no âmbito do paradigma sócio-crítico. Aborda o fundamento conceitual desta metodologia, seu escopo e os procedimentos usuais na perspectiva de seus representantes teóricos mais significativos. Investigar do paradigma sócio-crítico implica intrinsecamente um compromisso político, social, pela justiça, a equidade e a paz. A partir da história do PAP, verifica-se que este método implica a promoção de transformações sociais ou educacionais, para as quais é definido como um processo que não só busca compreender e gerar conhecimento, mas também espera despertar mudanças cultivadas por e com os atores envolvido Seus princípios exaltam a natureza indutiva, dialógica e ideográfica do conhecimento; Além disso, estabelecem uma relação inseparável entre teoria e prática. Os procedimentos propostos por diferentes autores sempre envolvem fases recursivas de ação-reflexão-ação, um ciclo aberto em movimento permanente. No final, veremos que esse tipo de pesquisa representa uma oportunidade para a transformação da cadeira de paz, que permitirá visualizar as possíveis aplicações do método no contexto universitário

Palavras-chave: geração de conhecimento, pesquisa de ação participativa, transformação social. 


\section{Presentación}

El presente artículo identifica las principales características del paradigma sociocrítico y del método de investigación acción participante (IAP), con la idea de justificar una alternativa para que la investigación en la universidad se ponga al servicio de las trasformaciones de los procesos sociopolíticos relacionados con la paz. La formación, de sujetos políticos y constructores de paz exige el uso de metodologías activas y participativas relacionadas con estas opciones epistemológicas; de allí que se presente un artículo de revisión teórica genérica acerca de la IAP $\mathrm{y}$, a la vez, se contextualice dicha revisión y se proyecte cómo podría operacionalizarse dicha opción metódica para abordar el objeto de conocimiento.

La Cátedra de Paz universitaria, es concebida como un espacio para la formación de sujetos políticos y constructores de paz que exige el uso de metodologías activas y participativas; en consecuencia, el fenómeno a estudiar exige la revisión de los principios fundamentales del paradigma sociocrítico y su relación con la IAP, además de una revisión sobre lo que significa conceptualmente este método, sus características y los procedimientos desde sus representantes teóricos más significativos.

Investigar desde el paradigma sociocrítico implica intrínsecamente un compromiso político y social por la justicia, la equidad y la paz. Desde la historia de la IAP se verá que este método implica la promoción de transformaciones sociales o educativas, por eso es definido conceptualmente, como un proceso que no solo busca comprender y generar conocimiento, también espera suscitar cambios gestados por y con los actores involucrados. Sus principios y características exaltan su naturaleza inductiva, dialógica, ideográfica y establecen la relación teoría-práctica. Asimismo, los procedimientos plantados por diferentes autores siempre implican fases recursivas de acción, reflexión acción. La cosmovisión y la metódica propuesta se consideran una unidad dialéctica entre lo teórico y lo práctico.

El origen del paradigma crítico se remonta desde Marx ala escuela de Frankfurt(Horkheimer, 1974; Adorno, 1975; Habermas 1986), quienes exponen la teoría cuyo objetivo era la emancipación del ser humano y es conocida como Teoría Crítica; sin embargo, también tiene soporte en una corriente latinoamericana representada por Freire (1981) y Orlando Fals Borda (1987). En general, surgen de una crítica a la racionalidad deductiva, instrumental y técnica glorificada por el paradigma positivista, plantean la necesidad de establecer una racionalidad particular que incluya las voces, los juicios, los valores y los intereses de los grupos implicados en las dinámicas sociales, así como sus compromisos para la transformación desde el seno de sus contextos (Bonilla y Rodríguez, 2013).

Esta propuesta procura superar el reduccionismo con que las ciencias sociales han abordado sus objetos de estudio, estableciendo una dicotomía insalvable entre los abordajes puramente empíricos y los interpretativos; así, eesta propuesta epistemológica surge de un compromiso social explícito, introduce la autorreflexión crítica en los procesos del conocimiento y pretende dar aportes que favorezcan el cambio social desde el interior de los propios contextos investigativos.

La IAP, inscrita en este paradigma, se propone transformar la estructura de las relaciones sociales y dar solución a determinadas situaciones, partiendo de un proceso de acción reflexión acción con los integrantes de la comunidad. En el ámbito educativo, propicia cambios en los aprendizajes y en las instituciones o contextos educativos que deben dar respuestas a esos procesos sociales.

\section{Paradigma sociocrítico}

Un paradigma es una entidad de creencias, principios, reglas y procedimientos que definen cómo hay que generar conocimiento; es el lente con que se mira al mundo, la cosmovisión de cada investigador de acuerdo con una perspectiva social e históricamente determinada (Kuhn, 1962; 1986; 1989; Lakatos, 1993a; 1993b).

Kuhn (1986) consideró a los paradigmas como realizaciones científicas universalmente reconocidas que, durante un tiempo determinado, proporcionan modelos a una comunidad científica, la cual desarrolla la ciencia dentro de un marco que se constituye de valores, conceptos y técnicas para orientar las actividades de los investigadores. Un paradigma designa una postura, una opción o un modo de investigar, un sistema conceptual expresado en un lenguaje, una opción que se expresa en vías técnico-instrumentales y que responden a un fondo filosófico o a una manera de ver el mundo.

El paradigma sociocrítico tiene sus orígenes en Marx y Engels en el siglo XIX, quienes hicieron más de lo que creyeron hacer en este sentido. Entre tantos aportes, resultan muy significativos: la tensión dialéctica, la reflexión crítica que intenta debelar las apariencias y la búsqueda de una sociedad emancipada y justa, lo que implica la interpretación del hombre y la historia. Este interés, encuentra sus seguidores en los críticos de la escuela de Frankfurt, quienes prosiguen los esfuerzos realizados por Korsch (1923) y Lukács (1923), lo cual desemboca en Habermas (1981) y el desarrollo de las teorías a las que se dedicó (Mardones, 1994; Leyva, 1999; Guba y Lincon 2002).

Este paradigma ha tenido diferentes realizaciones de acuerdo con los tantos seguidores, contextos y épocas. La libertad para ejercer la crítica busca identificar el potencial de cambio, por tanto, para evitar confusión e identificar los aspectos distintivos de este paradigma en la actualidad y contextualizados en Latinoamérica, Rubiano (2015) alega con Flecha (1997) que el pensamiento y la acción crítica deben definirse por lo que afirman más que por lo que niegan; de esta manera, una investigación será 
de naturaleza crítica si va tras un cambio social, si plantea una figuración del futuro que surge de la comprensión del presente, si promueve una actitud cuestionadora, si escudriña las contradicciones - "la crítica procede así desenmascarando ilusiones y apariencias" (Leyvay y Sampaio, 2012, p. 322) —, si se asume el problema de la subjetividad como un proceso de dar sentido, si forma parte de la realidad que desea conocer y modificar y si simultáneamente se transforma a sí mismo, si la teoría y práctica resultan inseparables, si los sujetos resultan protagonistas de sus formas de vida-, si defiende la justicia y la igualdad de todas las personas y sus colectivos, si apoya a los movimientos sociales que luchan por la igualdad, la justicia y la paz.

El paradigma sociocrítico tiene como objetivo trasformar la estructura de las relaciones sociales y promover cambios y avances, dando respuesta a problemas específicos presentes en el seno de los contextos sociales tales como, comunidades, movimientos sociales e instituciones, con la participación de sus miembros (Alvarado y García, 2008).

Este paradigma se fundamenta en la crítica social y plantea como condición el ser autoreflexivo. Supone que el conocimiento se construye por intereses que emergen de las necesidades de los grupos. Promueve que la voluntad racional y liberadora del ser humano puede alcanzarse si los sujetos se forman para la vida en común, si participan en la transformación social. Utiliza la autorreflexión y el conocimiento con el fin de que cada miembro tome conciencia del rol que le corresponde dentro del grupo.

El saber se hace a través de la construcción y reconstrucción continua de la teoría y la práctica, es decir, el hacer es un saber y el saber debe ser un hacer, es decir, debe tener una implicación práctica. De allí que sea necesario precisar que en este paradigma prevalece la disposición a un pensamiento inductivo, en el que las implicaciones teóricas derivan de la realidad estudiada, de la práctica y de la acción, de las cuales se resultan, a su vez, la reflexión y la sistematización. Se presentan en la Tabla 1 algunos principios del paradigma relacionados con este argumento.
Los procesos de participación se pueden dinamizar en toda comunidad como contexto sustancial para el trabajo social. En la búsqueda de soluciones es importante que se construyan acciones en la comunidad con una ocurrencia plurifactorial y multidisciplinaria, es decir, que todas las organizaciones políticas, educativas, sociales y los representantes de las instituciones de cada campo de conocimiento, no solo ayuden a resolver problemas sino que contribuyan a elevar la calidad de vida de sus integrantes o la calidad del desempeño particular de los integrantes en los diferentes escenarios, "El conocimiento se constituye siempre en base a intereses que han ido desarrollándose a partir de las necesidades naturales de la especie humana y que han sido configurados por las condiciones históricas y sociales" (Habermas, 1986, p. 169).

En el campo educativo este paradigma también ha tenido un papel preponderante. Los procesos educativos resultan complejos, intersubjetivos, tienden a reproducirse en procesos mecánicos, transmisivos y memorísticos, por lo cual para dinamizarlos y hacerlos pertinentes es necesario que logren implicarse en procesos transformadores que susciten cambios en las estructuras, los procedimientos, las estrategias de enseñanza y de evaluación, en el diálogo de saberes y en los aprendizajes cooperativos. Para el efecto, son necesarios recursos didácticos y espacios que favorezcan los aprendizajes y que fortalezcan las relaciones de los miembros que conforman los grupos.

Entre las características importantes del paradigma sociocrítico aplicado a la educación, se plantea una visión holística en la que cada parte implica el todo y se relaciona con las otras partes, democrática y participativa, por la que todos los participantes son importantes, sus puntos de vista y sus aportes, lo que conlleva a la aceptación y el respeto por la diferencia humana. Por último, supone una perspectiva inductiva lo que permite que el conocimiento emerja de la práctica (Figura 1).

Este paradigma se operacionaliza mediante la IAP; así, mientras el paradigma planea filosóficamente una forma de conocer, el método ofrece una guía que orienta, que propone una forma de conocer, claro está, aquí el

Tabla 1. Principios que ayudan a definir el paradigma sociocrítico

\author{
Tiene como objetivo central el análisis de las transformaciones sociales. \\ Busca dar respuestas a los problemas que se producen debido a las transformaciones sociales. \\ Enfatiza en el conocimiento y comprensión de la realidad como praxis. \\ Tiende a la unión de teoría y praxis, esta convergencia es entendida como: conocimiento, acción y valores. \\ Procura la emancipación del saber. \\ Se erige sobre la base de orientar el conocimiento para emancipar y liberar al hombre. \\ Involucra al investigador para que participe a través de la autorreflexión. \\ Se propicia la reflexión y crítica de los intereses, interrelaciones y prácticas educativas.
}

Fuente: Latorre, Rincón y Arnnal (1996). 

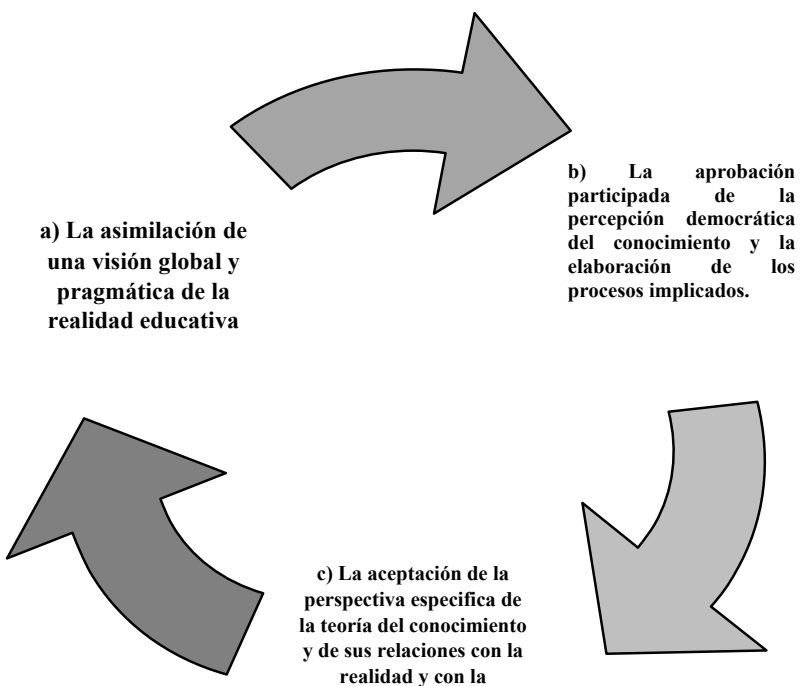

realidad y con la

práctica.

Figura 1. Características del paradigma sociocrítico aplicado a la educación

Fuente: Álvaro y García (2008, p. 191).

conocimiento se concibe en una relación indivisible presente, como ya se ha señalado, entre la teoría y la práctica.

El fenómeno a estudiar, relacionado con la necesidad de transformar y crear formas de promover la educación para la paz, demanda atención desde esta perspectiva, pues esta obligación socioeducativa deriva de graves problemas enquistados en la estructura de la sociedad colombiana que generan falta de equidad, injusticias, pobreza, focos de violencia enraizados en la historia; implica entonces la necesidad de que los jóvenes se impliquen no solo en la comprensión de los problemas que afectan la paz sino en las posibles soluciones que pueden aportar en sus contextos inmediatos.

\section{Método de la investigación acción participante}

A la IAP se le confiere su punto de origen en las investigaciones llevadas a cabo por Kurt Lewin en la década de 1940-1950, a raíz de la segunda guerra mundial, donde le solicitan investigar sobre cómo modificar los hábitos alimenticios de la población ante la escasez de determinados artículos. La intención de los estudios era resolver problemas prácticos y urgentes, donde los investigadores tenían que asumir el rol de agentes de cambio, en conjunto con la comunidad a intervenir. En estas circunstancias se empiezan a identificar y desarrollar las características de la investigación acción, tales como el conocimiento, la intervención, la mejora y la colaboración (Suárez, 2002, p. 40).

Lewin (1946) plantea rasgos cruciales de la investigación y acción:

La IAP es emprendida por personas, grupos o comunidades que llevan a cabo una actividad colectiva en bien de todos, consistente en una práctica reflexiva social en la que interactúan la teoría y la práctica con miras a establecer cambios apropiados en la situación estudiada y en la que no hay distinción entre lo que se investiga, quién investiga y el proceso de investigación (Restrepo, 2005, p. 1).

El andar histórico de la IAP ha trascurrido en dos perspectivas: la primera desde la sociología, cuyos representantes Kurt Lewin (1946), Chicago Sol Tax (1958) y Orlando Fals Borda (1970), entre otros, pretendieron transformaciones sociales. Esta metodología se fue abriendo paso en el mundo académico, dando lugar a la otra perspectiva relacionada con la educación, la cual fue inspirada por los pensamientos y prácticas de Paulo Freire (1974), Stenhouse (1985), John Elliott (1981; 1990) quien fue el seguidor de Stenhouse en Inglaterra, así como por Carr y Stephen Kemmis (1988) de la Universidad de Deakin en Australia. La IAP aplicada a la educación mantiene la unidad dialéctica entre la teoría y la praxis, cuyo desarrollo en diversos países mostró procesos de aprendizaje significativos y estableció una persistente relación innovadora entre los investigadores y los actores sociales.

La IAP en América Latina surgió a partir de los años 70 , en el ámbito de la modernización social; se introdujo en el desarrollo de la planificación social y educativa. En estos años, Fals Borda, Bonilla y Castillo (1972) proponen crear el centro de investigación y acción social, donde se produce la enunciación de la IAP como se le conoce actualmente.

Fals (1985), propone en su método una incorporación del investigador en la comunidad, la promoción de cambios en la conciencia de los miembros de la comunidad, la interpretación colectiva de los escenarios históricos y 
de la estructura social de la comunidad, así como la gestación de organizaciones políticas y grupos de acción por estas razones, se la llamó también investigación militante, caracterizada por su énfasis en la solución de problemas y el compromiso con la comunidad o grupo.

Las contribuciones teóricas de Lewin y Fals Borda se pueden "reinterpretar y actualizar complementariamente para aprobar y perfeccionar el conocimiento producido, convirtiéndolo en acción social en el plano comunitario" (Jiménez 1994, p. 104).

El concepto de la IAP es amplio, puesto que existen múltiples definiciones y no en todos los casos hay criterios unificados; no obstante, los diferentes enfoques coinciden en participar de los postulados de la epistemología crítica y orientar el análisis y la intervención como un aprendizaje constructivo y orientado a promover y lograr cambios sociales.

En síntesis, los conceptos de los diferentes autores pretenden mostrar que el IAP no termina en producción de conocimientos, sino que busca intervenir frente a las realidades sociales, transformándolas desde los participantes de la comunidad. Se parte de un problema advertido por todos, se hace crítica, pública, colectiva y planificada con el fin de mejorar la calidad de los procesos vividos.

Resulta importante destacar que el proceso es en sí mismo un medio y un fin, pues asumir una actitud dialógica, como bien lo han señalado, implica la fe en el otro, en el poder que tiene para transformar sus realidades; teniendo conciencia que si bien sus recursos, capacidades y fuerza de lucha pueden estar disminuidas por la destrucción de la subjetividad, la exclusión y la injusticia, se puede renacer gracias a la comprensión, a la participación que crea confianza en sí mismo y a la autorreflexión.

No es una preocupación principal la obtención de datos o la constatación de hechos de manera única y excluyente. Lo prioritario es la dialéctica que se establece en los agentes sociales, entre unos y otros, es decir la interacción continua entre reflexión y acción. Una visión pragmática del mundo social, donde lo fundamental es el diálogo constante con la realidad para intervenir en su transformación (Álvarez, Guerra y Sosa 1995, p. 160).

En conclusión, los distintos exponentes de la IAP proponen esta metodología como una combinación de la teoría y la praxis, tomar conciencia de la realidad que posea una población mediante un pensamiento inductivo; además, no solo se fundamenta en la construcción del conocimiento sino en intervenir frente a la realidad social potenciando los actores sociales como transformadores del cambio. Su lógica es ideográfica, que implica estudios profundos a pequeña escala en grupos o comunidades locales y le asiste una lógica dialógica porque implica la relación intersubjetiva y la confianza en el otro.

\section{Elementos y objetivos de la IAP}

Los variados enfoques de la IAP han mantenido tres elementos que son: "a) ser una metodología para el cambio; b) fomentar la participación y autodeterminación da las personas que la utilizan, y c) ser expresión de la rela-

Tabla 2. Definiciones de algunos representantes de las dos vertientes de la IAP

Paulo Freire (1974)

Fals Borda (1981)

Kemmis S. (1984)

Stenhouse L. (1984)

Elliott J. (1993)
El hombre dialógico tiene fe en los hombres antes de encontrarse frente a frente con ellos. Esta, sin embargo, no es una fe ingenua. El hombre dialógico que es crítico sabe que el poder de hacer, de crear, de transformar, es un poder de los hombres y sabe también que ellos pueden, enajenados en una situación concreta tener ese poder disminuido. Esta posibilidad, sin embargo, en vez de matar en el hombre dialógico su fe en los hombres se presenta ante él, por el contrario, como un desafío al cual puede responder. Está convencido de que este poder de hacer y transformar, si bien negado en ciertas situaciones, puede renacer. No gratuitamente sino en la lucha por su liberación. (Freire, 1974, p. 108)

La IAP permite determinar un problema que tiene su origen en la comunidad, la cual define, analiza, resuelve y transforma radicalmente la realidad social, con el fin de mejorar el nivel de las personas inmersas en esa realidad; además reconoce la participación plena y activa de la comunidad en la totalidad del proceso de investigación, con la idea de que el proceso pueda crear en la gente mayor confianza en sus propios recursos y la incite a desarrollar la confianza en sí misma. En este enfoque el investigador es partícipe y aprendiz comprometido en el proceso de investigación, y este proceso lo lleva más a la militancia que al desinterés. (Fals, 1981, p. 65)

Una forma de indagación autorreflexiva de los participantes en situaciones sociales (incluyendo las educativas) para mejorar la racionalidad y justicia de: a) sus propias prácticas sociales o educativas, b) la comprensión de dichas prácticas, y c) las situaciones (e instituciones) en que estas prácticas se realizan (Latorre, 2003, p.15)

Indagación sistemática y mantenida, planificada y autocrítica, que se halla sometida a crítica pública y a las comprobaciones empíricas en donde estas resulten adecuadas. (Garrido, 2006, p.113)

Un estudio de una situación social con el fin de mejorar la calidad de la acción dentro de la misma (Latorre, 2003, p.14)

Fuente: elaboración propia. 
ción dialéctica entre conocimiento y acción”. (Contreras, 2002, p. 10). Se considera que estos elementos son una herramienta útil de adquisición y de transformación de la realidad, apropiada para quienes no poseen esa competencia, lo que resulta muy importante debido a que estos elementos en sí mismos promueven la participación de todo aquel que tenga interés en acceder a esta metodología. La IAP, en su conjunto, se establece como un instrumento de estimulación y promoción humana, donde certifica la intervención activa y democrática de la comunidad con el propósito y la ejecución de sus programas y proyectos de desarrollo.

Es relevante tener en cuenta que en esta metodología las teorías no se demuestran ni son comprobadas por los investigadores, puesto que se describe la realidad mediante el procedimiento en el cual una población crea sus propias teorías y soluciones sobre sí misma. Es la sistematización de experiencia la que legitima el saber que derive y los criterios de cientificidad serán dados por la rigurosidad del trabajo, la dependencia de los datos, la posibilidad de ser transferible a grupos comunes sin ninguna pretensión de generalización y la credibilidad dada por la triangulación de instrumentos o de fuentes.

Si los elementos del método resultan cruciales, los objetivos mucho más porque de acuerdo con ellos se podrá evaluar la consistencia del método con el objeto de investigación, las problemáticas planteadas y las intencionalidades de la investigación. Según McKernan (2001) “el propósito de la investigación-acción en comparación con gran parte de la investigación tradicional o fundamental, es resolver problemas diarios e inmediatos acuciantes de los profesionales en ejercicio" (p. 24); sin embargo, los objetivos de la IAP podrían ir mucho más allá:

- Promover la producción colectiva del conocimiento rompiendo el monopolio del saber y la información, permitiendo que ambos se transformen en patrimonio de los grupos postergados.

- Promover el análisis colectivo en el ordenamiento de la información y en la utilización de que de ella puede hacerse.

- Promover el análisis crítico utilizando la información ordenada y clasificada a fin de determinar las raíces y causas de los problemas y las vías de solución para estos.

- Establecer relaciones entre los problemas individuales, colectivos, funcionales y estructurales, como parte de la búsqueda de soluciones colectivas a los problemas enfrentados (Contreras, 2002, p. 11).

Estos objetivos de la IAP, ajustados a la investigación particular que se desarrolle, deben ser conocidos por todos - los investigadores y la comunidad-; de esta manera, se constituye un proceso de investigación conjunta y una efectiva democratización del conocimiento. Como se mencionó anteriormente, cuando se utiliza la IAP como instrumento de estimulación y promoción humana, de generación de conciencia y de extensión del conocimiento, se debe promover la investigación colectiva.

\section{Características de la IAP}

Las características de la IAP son consistentes con los objetivos que se proponen. Kemmis y McTaggart (2005) plantean algunas características, afirmando que la IAP es:

- Social, en la medida en que estudia la relación entre la esfera individual y la social.

- Participativa, pues las personas deben trabajar para mejorar sus propias prácticas dentro de contexto social.

- Práctica y colaborativa cuando promueve que las personas examinen las prácticas sociales, conduciendo a mejorar las prácticas durante y después del proceso de investigación; además, al investigador no se le considera un experto, es un coinvestigador que investiga con y para la gente.

- Emancipatoria, pues el enfoque no es jerárquico sino simétrico, lo que hace que los participantes establezcan una relación de iguales en aportación de la investigación.

- Interpretativa porque los resultados dependen de las soluciones dadas e interpretadas por los participantes involucrados en la investigación.

- Crítica, pues contribuye a que las personas no solo busquen mejoras prácticas en el marco de las condiciones sociopolíticas dadas, sino que actúen como agentes de cambio críticos de esas condiciones.

- Reflexiva en la medida en que las personas transforman su práctica a través de la crítica y autocrítica, práctica, reflexión.

- Transformadora de teoría y práctica, pues desde una reflexión crítica y sus derivaciones. Esta metodología obliga a abordar la práctica diaria, a partir de cómo la entienden las personas participantes, para "explorar el potencial de diferentes perspectivas, teorías y discursos que deben ayudar a iluminar prácticas particulares y situaciones prácticas, como base para el desarrollo de comprensiones críticas e ideas sobre cómo deben ser transformadas las cosas" (Kemmis y McTaggart, 2005, p. 568).

La IAP es una metodología comprometida con la transformación de la organización y práctica educativa y social, se convierte en un proceso crítico de intervención y reflexión. Es un proceso de investigación de conocimiento, de práctica, de cambio, pretendiendo construir y formular alternativas de trasformación de las prácticas colectivas. 


\section{Procedimiento para realizar la IAP (etapas, fases, ciclos)}

El método de la IAP es la guía orientadora mediante la cual el investigador ajusta los pasos de la investigación de acuerdo con las circunstancias propias de cada investigación, lo anterior en compañía con todos los actores del proceso. El método tiene que ver con el cómo se investiga, cuáles son los momentos que hay que desarrollar, con qué técnicas e instrumentos se llevará cuenta del proceso, con cuáles se desarrollará la sistematización de la experiencia para saber qué saberes se derivan y qué hacer a continuación.

Los precursores del método de la IAP y los investigadores que utilizan esta metodología han propuesto variadas series de etapas, fases, pasos o ciclos, que tienen diferentes designaciones; sin embargo, su esencia es la misma:
- Se aborda una realidad desde el interior de ella.

- Se realiza un diagnóstico sobre la problemática detectada a partir de la reflexión de con todos los actores participantes.

- Se programa un plan de acción para abordar en forma conjunta estrategias de solución en forma conjunta.

- Se reflexiona sobre el proceso de ejecución del plan de acción.

- Se ajusta el plan y se vuelve a accionar, así sucesivamente hasta que se transforme la realidad.

- De este proceso activo, participativo e iterativo van emergiendo saberes productos de la sistematización.

Al respecto, es frecuentemente usado el triángulo de investigación-acción-formación propuesto por Kurt Lewin (Figura 2).

El método de trabajo de campo consecuente con este triángulo se presenta en la Figura 3.

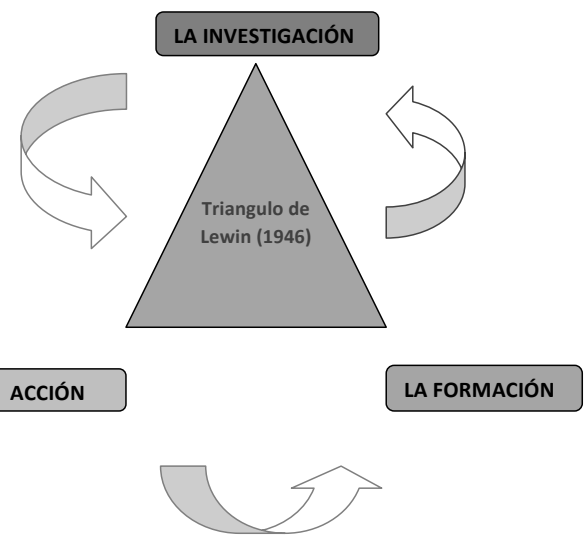

Figura 2. Proceso de la IAP

Fuente: Kurt Lewin (1946)

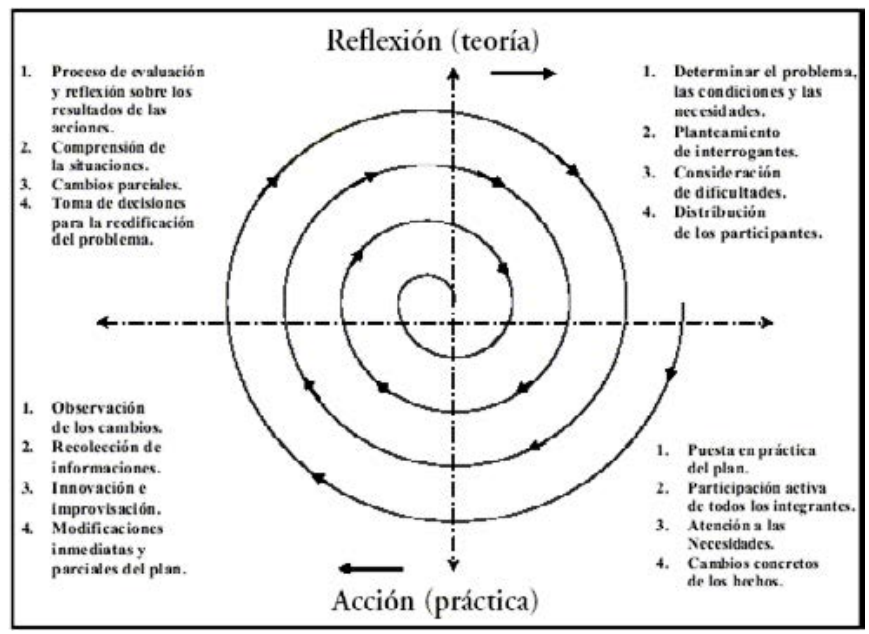

Fuente: Fontán (s.f.).

Figura 3. Método de la IAP 
Kemmis (1983) representa el proceso iterativo como se observa en la Figura 4, enfatizando la recursividad, que es un proceso crucial en este tipo de investigación.

El modelo espiral dialéctico planteado Kemmis y McTaggart (1992), resume el proceso en cinco aspectos: reflexión inicial-planificación-acción-observación-reflexión. Ahora bien, aunque en el proceso básico permanezcan varias etapas en común, vale la pena mirar los aspectos distintivos del método de la IAP que plantean los diferentes autores, ello también permite visualizar qué hacer en cada etapa (Tabla 3).

Existen diferentes referencias donde se describen las etapas, fases, ciclos, momentos y actividades, que se pueden seguir para realizar esta metodología IAP, la cual en todos los casos pueden variar dependiendo del proceso específico y los actores sociales involucrados; no obstante, en todos los casos se parte de un diagnóstico de la situación problema y la solución surge gradualmente de las acciones conjuntas.

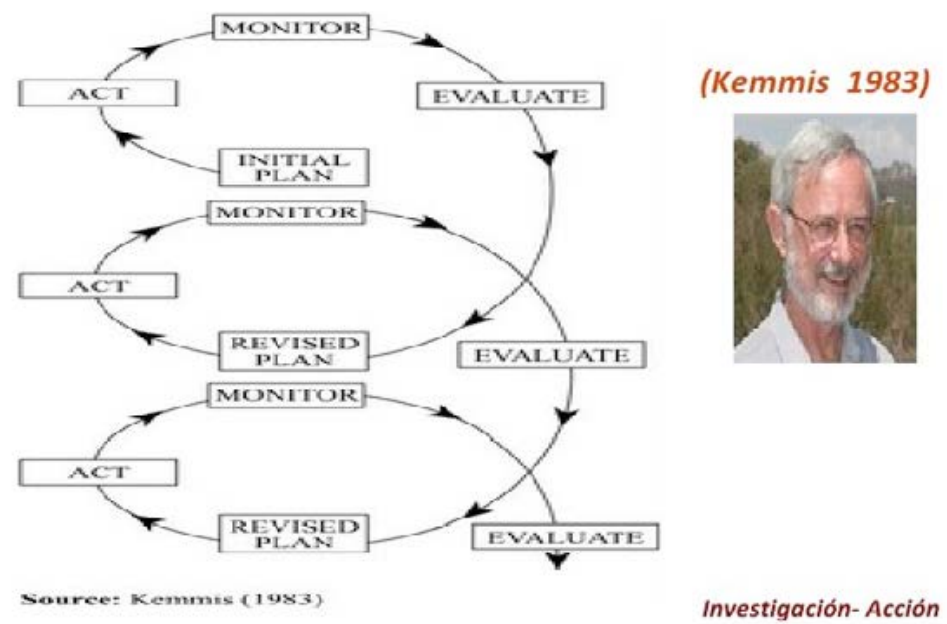

Fuente: Kemmis, S., (1983).

Figura 4. Método de la IAP

\section{Tabla 3. Algunos tipos procedimentales para aplicar la IAP}

\begin{tabular}{|c|c|}
\hline $\begin{array}{c}\text { Kemmis y McTaggart (1988) } \\
\text { Fases }\end{array}$ & $\begin{array}{l}\text { Organiza dos ejes, que denomina estratégico, que comprende acción y reflexión; y organizativo, que } \\
\text { implica la planificación y la observación, ambos incluidos en cuatro fases o momentos interrelacionados } \\
\text { e identificados como planificación, acción, observación y reflexión (Colmenares, 2011, p. 107). }\end{array}$ \\
\hline $\begin{array}{c}\text { Pérez (1990) } \\
\text { Pasos o etapas }\end{array}$ & $\begin{array}{l}\text { 1) Diagnóstico de una preocupación temática o problema. } \\
\text { 2) Construcción del plan de acción. } \\
\text { 3) La práctica del plan construido y su respectiva observación. } \\
\text { 4) Reflexión e interpretación de resultados y la replanificación si fuera necesaria (Pérez, 1990, p. 99). }\end{array}$ \\
\hline $\begin{array}{l}\text { Elliott (1993) } \\
\text { Actividades en forma } \\
\text { espiral }\end{array}$ & $\begin{array}{l}\text { 1) Identificación y aclaración de la idea general. } \\
\text { 2) Reconocimiento y revisión de los hechos de la situación. } \\
\text { 3)Estructuración del plan general. } \\
\text { 4) Desarrollo de las etapas de acción. } \\
\text { 5) Implementación de los siguientes pasos (Elliott, 1993, p.90). }\end{array}$ \\
\hline $\begin{array}{l}\text { Balcázar (2003) } \\
\text { Componentes }\end{array}$ & $\begin{array}{l}\text { 1) El grado de control que los individuos tienen sobre el proceso de investigación-acción. } \\
\text { 2) El grado de colaboración en la toma de decisiones que existe entre los investigadores. } \\
\text { 3) El nivel de compromiso de los participantes de la comunidad y los investigadores externos, con el } \\
\text { proceso de investigación y cambio social (Balcázar, 2003, p. 65). }\end{array}$ \\
\hline $\begin{array}{l}\text { Teppa, }(2006) \\
\text { Momentos }\end{array}$ & $\begin{array}{l}\text { 1) Inducción: diagnóstico. } \\
\text { 2) Elaboración del plan: planificación. } \\
\text { 3) Ejecución del plan: observación, acción, producción intelectual, reflexión. } \\
\text { 4) Transformación: replanificación (Teppa, 2006, p. 15). }\end{array}$ \\
\hline $\begin{array}{c}\text { Colmenares (2011) } \\
\text { Fases }\end{array}$ & $\begin{array}{l}\text { 1) Descubrir la temática. } \\
\text { 2) Coconstrucción del Plan de Acción por seguir en la investigación. } \\
\text { 3) Ejecución del Plan de Acción. } \\
\text { 4) Cierre de la Investigación, en la cual se sistematizan, categorizan y generan aproximaciones teóricas } \\
\text { que pueden servir de orientación para nuevos ciclos de la investigación (Colmenares, 2011, p. 107). }\end{array}$ \\
\hline
\end{tabular}

Fuente: elaboración propia. 
Constituye un proceso continuo, una espiral, donde se van dando los momentos: problematización, diagnóstico, diseño de una propuesta de cambio, aplicación de la propuesta, ajustes y valoración, para luego reiniciar un nuevo circuito partiendo de una nueva problematización. Estas fases del método son flexibles, permiten abordar los hechos sociales como dinámicos y cambiantes, por lo tanto, están sujetas a los cambios que el mismo proceso genere.

La información recolectada se debe contrastar usando técnicas de triangulación de las fuentes, instrumentos y los datos, ello asegura la credibilidad, la participación y la contrastación de la metódica, de los investigadores y la teoría.

El informe final debe ser de carácter descriptivo-narrativo-reflexivo y los resultados derivarán de la posibilidad de encontrar el sentido de los datos cualitativos. En principio forma parte de un proceso analítico el grueso de los datos condensados en unidades analizables, se crean categorías codificadas para poder manejar con más eficiencia los datos; posteriormente, se realiza el proceso de síntesis en el que se agrupan las categorías analíticas, se relacionan, se sucede una reducción de los datos. De esta manera, se podría decir con Coffey y Atkinson (2003) que el procedimiento de la información recopilada durante todo el proceso a través de las técnicas de observación participante, entrevistas individuales o colectivas, los grupos focales, de la revisión de documentos generados del proceso, de los registros minuciosos del desarrollo de las etapas, es el siguiente:

- Etiquetar segmentos de textos con información sobre la categoría a la cual pertenece.

- Los datos segmentados se codifican y clasifican en categorías y subcategorías.

- Recontextualización de los segmentos de datos.

- Se vinculan todos los fragmentos de los datos a una idea o concepto particular.

Otra condición importante para dar soporte a la reflexión es la controversia. La reunión y sistematización de la información se establece en los ejes que atraviesan todo el desarrollo del proceso, frente a ella el investigador genera oportunidades de controversia y de evaluación de los éxitos y los obstáculos, con el fin de tomar decisiones sobre la ruta a seguir y los comportamientos de cada uno de los actores en forma individual y colectiva, produciendo de este modo conocimiento surgido de la acción reflexionada y valorada. De este tipo de investigación emergen resultados relacionados con un antes, un durante y un después que puede ser un siguiente punto de partida.

\section{Punto de partida para la trasformación de la Cátedra de Paz en el ámbito universitario}

Ahora bien, una vez culminada la revisión teórica puede resultar interesante visualizar o vislumbrar parte del en- granaje teórico del método en una investigación concreta dada en el ámbito universitario.

La necesidad de abogar por la paz, dada la conflictividad de los procesos sociopolíticos en Colombia, representa la razón de este estudio. Desde una perspectiva crítica, bajo la metódica de una investigación acción participante, se asumirá La Cátedra de Paz como un espacio para la construcción de sujetos políticos y de paz con el uso de metodologías activas y participativas. Las técnicas de recolección de la información prevista son: grupos focales, talleres y círculos de acción reflexión acción y a partir de allí participarán y transformarán la cátedra de paz.

El paradigma crítico, resultará ser el más propicio para esta investigación, porque le da sentido a un fenómeno social en su medio natural, es decir, "se interesa por los significados que los participantes le dan a cada situación y estos significados solo pueden ser leídos en el contexto de la interacción de los individuos" (Filstead, 1986, p. 53). Además, busca interpretar el sentido que las personas y los colectivos le otorgan a la acción, a la vida cotidiana y a la construcción de la realidad social (Deslauriers, 2005); además, pretende suscitar transformaciones.

Se asumirá la metodología de investigación acción participativa (IAP) generada a partir de la indagación de las representaciones sociales de los estudiantes universitarios, comenzando con un proceso de acción-reflexión-acción irán modificando sus ideas previas, acciones y a la vez la estructura de la cátedra de la paz, para que justamente esté al servicio de la reflexión, configuración y reconfiguración de las concepciones y prácticas de los jóvenes respecto a la paz. Sus técnicas de investigación, como ya se ha justificado ampliamente, reconocen las bases sociales del conocimiento y a su vez el proceso de construcción y trasformación en las personas y grupos sociales.

El trabajo se realizará con estudiantes de la Universidad los Libertadores porque son ellos mismos los que pueden describir y conocer mejor sus propias representaciones sociales de paz, son también los artífices de los cambios en esas representaciones; se conformará un grupo de investigación con estudiantes de la Facultad de Psicología, de la asignatura Psicología y Educación, se tiene previsto, en principio, conformar un grupo motor de investigación-acción-participativa (GMIAP) de siete jóvenes universitarios conformados por dos hombres y cinco mujeres, con edades que oscilan entre los dieciocho y los veinticinco años de edad. Para los grupos focales y la aplicación de los talleres, se conformarán grupos de diez a doce estudiantes pertenecientes a la Facultad de Psicología con edades entre los dieciocho y los veinticinco años de edad.

En la IAP resulta imprescindible el reconocimiento del sujeto que se constituye al interior de los procesos participativos; en este sentido, para Fals (1981) la IAP asiente en establecer un problema que tiene su origen en la comunidad, en este caso en las representaciones sociales de 
los jóvenes, el cual precisa, analiza, resuelve y transforma radicalmente la realidad social, atendiendo a la particularidad de este estudio, estamos hablando de la cátedra de formación por la paz, con el fin de mejorar a las personas inmersas en esa realidad y, a su vez, propiciar adelantos en relación con la paz desde una relación inherente entre la teoría y la práctica.

En la primera fase se realizarán una serie de reuniones de trabajo con los estudiantes de la Fundación Universitaria los Libertadores, los cuales conformarían el grupo motor de investigación acción participativa (GMIAP) para apoyar el proceso de investigación; el grupo de estudiantes seleccionados será de la asignatura Psicología y Educación, se conforman así los grupos focales en los que se desarrollarán las entrevistas colectivas, las cuales explorarán las representaciones sociales de los jóvenes en torno a la paz.

En esta fase se realizará: (a) La conformación del grupo motor de investigación acción participativa (GMIAP), se dará a conocer el objetivo del proyecto y se propicia la participación activa durante el proceso; (b) el grupo GMIAP generará la participación de los compañeros universitarios para conformar un grupo focal (entre diez y doce estudiantes) para indagar sobre las representaciones sociales de paz, recolectar la información necesaria y contextualizar el conocimiento producido para obtener el diagnóstico; (c) posteriormente, se hará otro grupo focal con los sujetos de la investigación para desarrollar el diagnóstico.

Se elaborará el análisis específico de las representaciones sociales de paz que tienen los estudiantes universitarios obtenidas del primer grupo focal, las cuales servirán como punto de partida para poner en funcionamiento el plan de acción. El grupo motor de investigación acción participativa (GMIAP) será un grupo de apoyo, auxiliares de investigación que tendrán la oportunidad de reflexionar acerca de sus propias representaciones sociales al igual que las de la investigadora.

En una segunda fase se realizará una serie de talleres para que estos estudiantes cambien su papel de informantes y se transformen en actores clave del proceso de investigación, diagnostiquen sus representaciones sociales, participen en la construcción y ejecución del plan de acción relacionado con la cátedra de paz.

Se presentan a continuación los pasos a ser ejecutados durante los talleres: (a) con el grupo motor de investigación acción participativa (GMIAP) se hará un diagnóstico procurando organizar y ejecutar el trabajo de campo; (b) se aplicarán tres talleres donde se trabajarán las representaciones sociales de paz, por otro lado, a través de actividades como asociaciones de palabras, obtención de fotografías y elaboración de cuentos diseñados por los jóvenes se obtendrá información de la temática a partir de la cual se busca generar una propuesta para la transformación de la cátedra. Se realizará un registro riguroso de esta fase para poder cumplir con el proceso de acción-reflexión-acción como un proceso recursivo.

En una tercera fase, los estudiantes podrán sistematizar y valorar a través de los Círculos de Reflexión las trasformaciones suscitadas de las RS sobre paz con la participación de todos los participantes de la investigación IAP y las propuestas hechas para la cátedra. Esta fase corresponde a la fase evaluativa: se valoran los materiales, técnicas y estrategias utilizadas por parte de todos los participantes en el proceso de IAP, se diseña un formato de evaluación de los talleres y se transcribe la información de los talleres y grupo focal, se revisan las anotaciones, fotografías, grabaciones de sonido o videos de las acciones o eventos que emergen de la investigación. Luego se utiliza el software Nvivo 11 para el análisis cualitativo de los datos, se sistematiza la información y se teoriza para dar cumplimiento al objetivo general.

El método será un proceso simultáneo y continuo fundamentado en la investigación-acción-formación, con la intención de poder hacer un cambio de las representaciones sociales de los jóvenes, de la cátedra y de los espacios de reflexión relacionados con la realidad de las prácticas cotidianas de los estudiantes universitarios. La IAP permite que los investigados se conviertan en actores de la investigación, que en la medida en que adquieren conocimiento sobre su realidad identifiquen los caminos para transformarla. Lo anterior es perfectamente coherente con el objetivo de la investigación que busca estructurar una cátedra de paz dirigida por los propios estudiantes, capaz de transformar sus prácticas cotidianas y generar su aporte a la construcción de la paz. Esto se debe a que la IAP, rompe con la verticalidad entre los actores involucrados, el individuo no es solamente el objeto de estudio, sino que este, después del planteamiento del lugar de investigación, se convierte en uno más de los entes de investigación con derechos de aportar, opinar, hacer cuestionamientos y planteamientos para la solución de problemas.

Por tanto, este método permite comprender a la juventud universitaria participante como sujeto (no como objeto) teniendo en cuenta sus habilidades, potencialidades, experiencias, conocimientos, representaciones que los faculta a contribuir a través de la cátedra de paz a que construyan una cultura de paz y ser protagonistas de este estudio.

Es importante tener en cuenta en la estructura de la IAP que este tipo de investigación varía según el entorno, tiempo, lugar y el interés de las personas de estudio. Estos momentos serán recurrentes, abiertos y flexibles. Los instrumentos de recolección de datos serían, en un primer momento, el grupo focal que tendría como propósito saber cuáles son las representaciones de los jóvenes, es decir, lo qué conocen y piensan los estudiantes universitarios sobre lo que es la paz y así poder identificar cuáles son los temas que se deben trabajar en los talleres. 
En un segundo momento el taller será una actividad grupal, orientada hacia el conocimiento práctico y el análisis relativo al diagnóstico de las representaciones sociales de paz que se hayan obtenido en la aplicación de los grupos focales. Con base en el diagnóstico, el grupo GMIAP socializa con el grupo de diez a doce estudiantes de la Facultad de Psicología los temas que se tratarán en los talleres. La intención es transformar sus representaciones sociales sobre la paz, que se transformen en constructores de paz y, de igual forma, transformar la cátedra de la paz.

El círculo de reflexión, abordará los temas tratados en los talleres y, si se logra, una trasformación de las representaciones sociales de paz en los participantes, los posibles temas que deben incluirse en la cátedra de paz y las actividades que se pueden seguir haciendo para crear una cultura de paz entre los universitarios. Se plantea la elaboración del syllabus de la cátedra de paz para proponerlo a la universidad.

Una vez que se obtenga la información de la aplicación de las técnicas del grupo focal y taller, se realizará una codificación a través de palabras clave que se asignarán a cada respuesta, las cuales se agrupan considerando las categorías y subcategorías utilizando el programa de análisis cualitativo Nvivo 11.

El análisis se hará a través de la sistematización de experiencias, el cual está vinculado con el método IAP, donde las reflexiones colectivas de los estudiantes involucrados se autoinvestigan para comprender y generar activamente cambios institucionales o sociales; además, se presenta la información con el fin de que los universitarios analicen y planteen sus propuestas de acción y se logre rediseñar la cátedra de paz. Luego que la información esté trascrita, categorizada y codificada, es necesario cerrar con la reducción de datos llevada por un proceso de síntesis e interpretación, el cual dará paso a la propuesta que se pretende elaborar.

\section{Consideraciones finales}

Se puede utilizar la IAP para generar procesos de cambio debido a que es una metodología que respalda la producción de un conocimiento objetivo y transformador, generado mediante la discusión, reflexión y cimentación colectiva de los distintos saberes de las personas de la comunidad en el contexto de un proceso para lograr una trasformación social. De esta manera, interesa la IAP en el campo educativo para favorecer la comunicación, el intercambio, el diálogo, la participación entre iguales utilizando como instrumento de construcción de sí mismos, de poder democrático y del progreso de su comunidad. Asimismo, estimula la reflexión y autoreflexión subjetiva y colectiva, la toma de conciencia, la actitud ética y el compromiso político, en este caso en torno a la construcción de la paz.
Con la IAP se busca que las personas tiendan a ser actores de su propio comportamiento y puedan elegir libremente las decisiones frente a su desarrollo y el origen de su propio destino. En esta metodología se busca desarrollar acciones concretas de intervención que se queden en el presente, pero tengan incidencia en el futuro.

No hay que dejar de lado el papel del investigador que, al ser parte de la IAP, también se fortalece de ese proceso de empoderamiento y de concientización que experimenta al lado de las personas participantes de la comunidad, haciendo que sus representaciones, sus comportamientos se transformen y tengan nuevas comprensiones ante la sociedad con la que interactúa.

Ahora bien, la participación en la construcción de paz resultará de suma importancia, las personas que forman parte de una comunidad tienen la capacidad y el compromiso para ser sujetos dinámicos en su desarrollo, en la observación y reflexión de sus propias dificultades y en la búsqueda de soluciones creativas y eficaces. He allí la razón de haber escudriñado las definiciones, elementos, características, objetivos y los procedimientos de la IAP, y de haber precisado su valor en el campo educativo; ello podrá contribuir a la construcción de una propuesta de para la Cátedra de Paz a partir del proceso participativo de los jóvenes universitarios que los ponga a compartir saberes, creencias, sus representaciones sociales, experiencias previas y los coloque en interlocución con otros jóvenes, buscando con ello generar compromiso, empoderamiento y transformación para la construcción de la paz.

La necesidad de abogar por la paz, dada la conflictividad de los procesos sociopolíticos en Colombia, representa la razón de haber revisado el paradigma sociocrítico y la IAP en este artículo; desde esta perspectiva se asumirá en forma colectiva la Cátedra de Paz como un espacio para la construcción de sujetos políticos y de paz con el uso de metodologías activas y participativas en la universidad.

En conclusión, esta opción epistemológica y método de investigación resulta de gran utilidad para los estudios sociales y educativos; en este caso, se ha puesto al servicio de la educación universitaria y de la paz, procesos que ameritan del pensamiento crítico y de transformaciones permanentes.

\section{Referencias}

Adorno, T. (1975). Dialéctica negativa. Madrid: Taurus.

Alvarado L., y García M. (2008). Características más relevantes del paradigma soci-crítico: su aplicación en investigaciones de educación ambiental y de enseñanza de las ciencias realizadas en el Doctorado de Educación del Instituto Pedagógico de Caracas. Sapiens, 9(2), 187-202.

Álvarez, M. Guerra, C. y Sosa N., (1995). Investigación-acción participativa en la periferia urbana de Salamanca. Cuadernos de la Red, 3, 156-173 
Balcázar, F. (2003). Investigación acción participativa (IAP): Aspectos conceptuales y dificultades de la implementación. Fundamentos en humanidades, 4(7/8), 59-77.

Bonilla, E., y Rodríguez, P. (2013). Más allá del dilema de los métodos. La investigación de ciencias sociales. Bogotá D.C.: Universidad de los Andes.

Callejo, J. y Viedma, A. (2006). Proyectos y estrategias de investigación social: la perspectiva de intervención. Madrid: McGraw-Hill.

Carr, W. y Kemmis, S. (1988). Teoría critica de la enseñanza. Barcelona: Ediciones Martínez Roca.

Colmenares, A. (2011). Investigación-acción-participativa: metodología integradora del conocimiento y la acción. Voces y silencios. Latinoamericana de Educación, 3(1), 102-115.

Coffey, A. y Atkinson, P. (2003). Encontrar el sentido a los datos cualitativos. Medellín: Editorial Universidad de Antioquia.

Contreras, R. (2002) La investigación Acción Participativa (IAP): revisando sus metodologías y sus potencialidades. En J. Durston y F. Miranda (Ed.). Experiencias y metodología de la investigación. (pp. 58- 71). Santiago de Chile: CEPAL.

Deslauriers, J. (2005) Investigación cualitativa. Pereira: Papiro

De la Garza, T. y Leyva, G. (2012). Tratado de la metodología de las Ciencias Sociales: perspectivas actuales. Ciudad de México: Universidad Autónoma Metropolitana.

Elliott, J. (1981). A framework for self-evaluation in schools. Sur África: University of Cape Town.

Elliott, J. (1990). La investigación-acción en educación. Madrid: Morata.

Elliott, J. (1993). El cambio educativo desde la investigación acción. Madrid: Morata.

Fals, O. (1985). Conocimiento y poder popular. Bogotá, Colombia: Siglo XXI Editores.

Fals, O. (1970). Ciencia propia y colonialismo intelectual. México: Nuestro Tiempo.

Fals, O., (1981). Historia doble de la Costa: Tomo II, El Presidente Nieto. Bogotá D.C.: Carlos Valencia Editores.

Fals, O. (1985). Conocimiento y poder popular. Bogotá D.C.: Siglo XXI Editores.

Fals, O. (1987). Ciencia propia y colonialismo intelectual. Los nuevos rumbos, Bogotá D.C., Colombia: Carlos Valencia Editores.

Fals, O., Bonilla, V., y Castillo, G. (1972). Causa popular, ciencia popular. Bogotá D.C.: Publicaciones de La Rosca.

Fals, O., [et .al.], (1981). Investigación participativa y praxis rural, nuevos conceptos en educación y desarrollo comunal. Lima: Mosca Azul Editores.

Filstead, W. (1986). Métodos cualitativos. Una experiencia necesaria en la investigación evaluativa. En T. Cook y C. Reichar (Eds.), Métodos cualitativos y cuantitativos en investigación evaluativa. Madrid: Ediciones Morata.

Fontán, M. (s.f.). Investigación-acción. Madrid: Universidad de Navarra.
Freire, P. (1974). Pedagogía del oprimido. Madrid: Siglo XXI Editores.

Freire, P. (1981). La educación como práctica de la libertad. Ciudad de México: Siglo XXI Editores.

Flecha, J. (1997). Pensamiento y acción crítica en la sociedad de la información. En J. Goikoetxea y J. García (Comp.), Ensayos de pedagogía critica. (pp. 29-43). Madrid: Educación Popular.

Garrido, F. (2006). Perspectiva y prácticas de educación-investigación participativa. Revista politica y sociedad, 44(1), 107-124.

Guba, E. y Lincon Y. (2002). Paradigmas en competencia en la investigación cualitativa. En C. Denman y J. Haro (Eds.). Por los rincones. Antropología de métodos cualitativos en la investigación social. (pp. 113-145). México: El Colegio de Sonora.

Habermas, J. (1981). Teoría de la Acción Comunicativa. Madrid: Taurus.

Habermas, J. (1986). Conocimientos e interés en ciencia y técnica como ideología. Madrid: Tecnos.

Horkheimer, M. (1974) Teoría tradicional y teoría crítica, en Horkheimer, Max Teoría crítica, Buenos Aires: Amorrortu.

Jiménez, B. (1994). Investigación ante acción participante: Una dimensión desconocida. En M. Montero. (Ed). Psicología Social Comunitaria: Teoria método y experiencia. (pp.103-137). Guadalajara: Universidad de Guadalajara.

Kemmis, S. (1983). Action research, En T. Husen and T. Postleth-Waite (Eds.). International encyclopedia of education: research and studies. Oxford: Pergamon.

Kemmis, S. (1984). Point-by-point guide to action research. Burwood: Victoria.

Kemmis, S. y McTaggart, R., (1992) ¿Cómo planificar la investigaciónacción? Barcelona: Laertes.

Kemmis, S. y McTaggart, R. (2005). Participatory Action Research. En N. Denzin y S. Lincoln (Eds.), The Sage Handbook of Qualitative Research. (pp. 559-603). Londres: Sage.

Korsch, K. (1923). Marxismus und Philosophie. Frankfurt am Main: Europäische Verlagsanstalt,

Kuhn, T. (1962). La estructura de las revoluciones cientificas. Buenos Aires: Fondo de Cultura Económica.

Kuhn, T. (1986). La estructura de las revoluciones cientificas. México: Fondo de Cultura Económica.

Kuhn, T. (1989). ¿Quéson las revoluciones cientificas? Barcelona: Paidós.

Kurt, L. (1946). Resolving social conflicts. Nueva York: Harper.

Lakatos, I. (1993a). La metodología de los programas de investigación cientifica. Madrid: Alianza.

Lakatos, I. (1993b). Historia de la ciencia y sus reconstrucciones racionales. Madrid: Tecnos.

Latorre, A., Rincón, D. y Arnnal, J. (1996). Bases metodológicas de la investigación educativa. Barcelona: Hurtado Mompeo Editor

Latorre, A., (2003). La Investigación Acción. Conocer y cambiar la práctica educativa. Madrid: Graó.

Lewin, K. (1946). Resolving social conflicts. Nueva York: Harper. 
Leyva, G. y Sampaio, M. (2012). Teoría crítica: El indisoluble vínculo entre la teoría social y la crítica normativa inmanente. En J. Goikoetxea y J. García (Comp.). Ensayos de pedagogía critica. Madrid: Educación Popular.

Lukács, G. (1923). Geschichte und Klassenbewußtsein. Berlin: Luchterhand, Darmstadt-Neuwied.

Mardones, J. (1994) Filosofia de las ciencias sociales, humanas y sociales. Materiales para la fundamentación cientifica. Barcelona: Anthropos.

McKernan, J. (2001). Investigación-acción y curriculum. Métodos y recursos para profesionales reflexivos. Madrid: Morata.

Ozanne, J. and Saatcioglu, B. (2008). Participatory action research. Journal of Consumer Research, 5(3), 423-439. https://doi. org $/ 10.1086 / 586911$

Pérez, G. (1990). Investigación-acción: aplicaciones al campo social yeducativo. Madrid: Dykinson.

Restrepo, G. (2005). Una variante pedagógica de la Investigación-Acción educativa. OEI-Revista Iberoamericana de Educación. Recuperado de http://www.rieoei.org/deloslectores/370Restrepo.PDF
Rubiano, E. (2015) Crítica a los planteamientos epistemológicos empleados en la investigación social. En A. Carosio (Coord.), Tiempos para pensar. Investigación social y humanistica hoyen Venezuela. (pp. 127-137). Caracas: Consejo Latinoamericano de Ciencias Sociales (CLACSO)

Salazar, M. (Coord.). (1992). La investigación-acción participativa. Inicios y Desarrollos. (pp. 27-36). Colombia: Editorial Popular.

Stenhouse, L. (1984). Investigación y desarrollo del curriculum. Madrid: Ediciones Morata.

Stenhouse, L. (1985), La investigación como base de la enseñanza, Madrid, España: Ediciones Morata

Suárez, M. (2002). Algunas reflexiones sobre la investigación acción colaboradora en la educación. Revista Electrónica de enseñanza de las Ciencias. 1(1).

Teppa, S. (2006). Investigación Acción participativa en la praxis pedagógica diaria. Barquisimeto. UPEL-IPB. 